\title{
The Applicability of Autosomal Short Tandem Repeats Genotyping for Minor Contributor DNA Profiling from Mixed Saliva Samples
}

\author{
Gabriela Repiská1*, Jaroslava Durdiaková2 ${ }^{\text {, Natália Kamodyová1 and Gabriel Minárik }}{ }^{1,3,4}$ \\ 1 Institute of Molecular Biomedicine, Comenius University Faculty of Medicine, Sasinkova 4, Bratislava, Slovakia \\ ${ }^{2}$ Institute of Physiology, Comenius University in Bratislava Faculty of Medicine, Sasinkova 2, Bratislava, Slovakia \\ ${ }^{3}$ Department of Molecular Biology, Comenius University in Bratislava Faculty of Natural Sciences, Mlynská dolina, Bratislava, Slovakia \\ ${ }^{4}$ GENETON Ltd, Cabanova 14, Bratislava, Slovakia
}

\begin{abstract}
Objective: In our previous study focused on Y-chromosome sequence identification and genotyping we revealed the possibility of male minor fraction identification and genotyping in mixed salivary samples obtained from females 60 and 30 minutes after intense kissing. The aim of this study was to test the applicability of an autosomal STR (aSTR) profiling kit for male fraction detection and genotyping on salivary samples obtained from females $1-60$ minutes after intense kissing.
\end{abstract}

Methods: The aSTR typing was performed on DNA samples originated from buccal swab and saliva samples collected from 12 heterosexual pairs before and after 2 minutes of intense kissing, respectively. The success of minor contributor allele identification was quantified as the ratio between Counts of Identified Obligatory Alleles and Counts of Potentially Identifiable Obligatory Alleles. For the estimation of proportion of minor contributor DNA the Y/X Amelogenin peak height ratio was used.

Results: In samples collected immediately after kissing has stopped the Amelogenin $\mathrm{Y} / \mathrm{X}$ signal ratio varied between 0 and $63 \%$. The ratio was associated with aSTR profiling success as in samples with higher than $7 \% \mathrm{Y} / \mathrm{X}$ ratio more than $80 \%$ of minor contributor male alleles were identified. In one sample collected 5 minutes after kissing expected male signal was detected with $\mathrm{Y} / \mathrm{X}$ ratio reaching $15 \%$ and $77 \%$ of obligatory male alleles were identified. In comparison of previously and currently utilized methods for minor contributor male DNA detection and identification the concordance in their performance was recorded.

Conclusion: We confirmed that in salivary mixtures, female saliva DNA analysis with use of aSTR genotyping kit is possible, but with limited effectiveness. The male admixture was detected with aSTR genotyping kit in salivary samples collected up to 5 minutes after intense kissing.

Keywords: Forensic science; DNA analysis; Short tandem repeat profiling; Mixed sample analysis; Sexual crime evidence; Constrained kissing; Saliva samples

\section{Introduction}

In forensic analysis, specimens from the crime scene are mostly represented by two-person mixtures, especially in cases of sexual assault investigation $[1,2]$. The most analysed type of sample mixture after sexual assault are male/female DNA mixed samples. This mixture is composed of predominantly victim DNA (mostly of female origin); the perpetrator's DNA (mostly of male origin) is in minority [3]. If the ratio of the minor contributor DNA is smaller than $1: 10$, it is currently challenging to obtain a conventional autosomal DNA profile of the minor contributor, with consequential loss of potentially crucial DNA evidence. In such cases, Y-chromosomal short tandem repeats (Y-STR) profiling is of great help, because it is possible to successfully profile the male Y-STR haplotype that could be used for identification of the perpetrator $[4,5]$. However, Y-STR profiling has insufficient discrimination power and is usually not widely used for individual identification [6], although a recent study focused on ultrahigh discrimination multiplex DNA typing based on Y-STR profiling showed its applicability for personal identification [7]. Satisfactory resolution is typical for widely used autosomal STR (a-STR) profiling kits. Recently, commercially available a-STR profiling kits have evolved and are able to successfully determine DNA profiles from mixtures with less than $5 \%$ of the minor component [8]. To test if this level of minor component identification could be sufficient for use in forensic analysis, artificially prepared samples that mimic forensic samples are commonly analysed. During acts of sexual violence, constrained kissing often occurs and the perpetrator's saliva is mixed with that of the victim. This could be potentially used to identify the perpetrator's DNA profile from the victim's saliva [9]. Of course, in salivary samples the foreign DNA remains detectable only for a limited time because of the victim's continuing salivation (if the victim survived the attack) and therefore it is necessary to collect the sample in a relevant time after the act. In our previous study, we confirmed that the male DNA persisted in a woman's saliva at detectable levels for at least 60 minutes in $67 \%$ of samples. Moreover, it was possible to obtain the full Y-STR profile even in salivary samples collected 30 minutes after the intensive kissing had stopped [10]. The aim of the current study was to test the possibility of using salivary samples collected from women at given intervals after intense kissing for detection of the minor contributor

*Corresponding author: Gabriela Repiská, Institute of Molecular Biomedicine Comenius University in Bratislava Faculty of Medicine, Sasinkova 4, 81108 Bratislava, Slovakia, Tel: +421-2-59357371; Fax: +421-2-59357631; Email: gabika.repiska@gmail.com

Received October 10, 2014; Accepted December 04, 2014; Published December 10, 2014

Citation: Repiská G, Durdiaková J, Kamodyová N, Minárik G (2015) The Applicability of Autosomal Short Tandem Repeats Genotyping for Minor Contributo DNA Profiling from Mixed Saliva Samples. J Forensic Res 6: 259. doi:10.4172/2157 7145.1000259

Copyright: (c) 2015 Repiská G, et al. This is an open-access article distributed under the terms of the Creative Commons Attribution License, which permits unrestricted use, distribution, and reproduction in any medium, provided the original author and source are credited. 

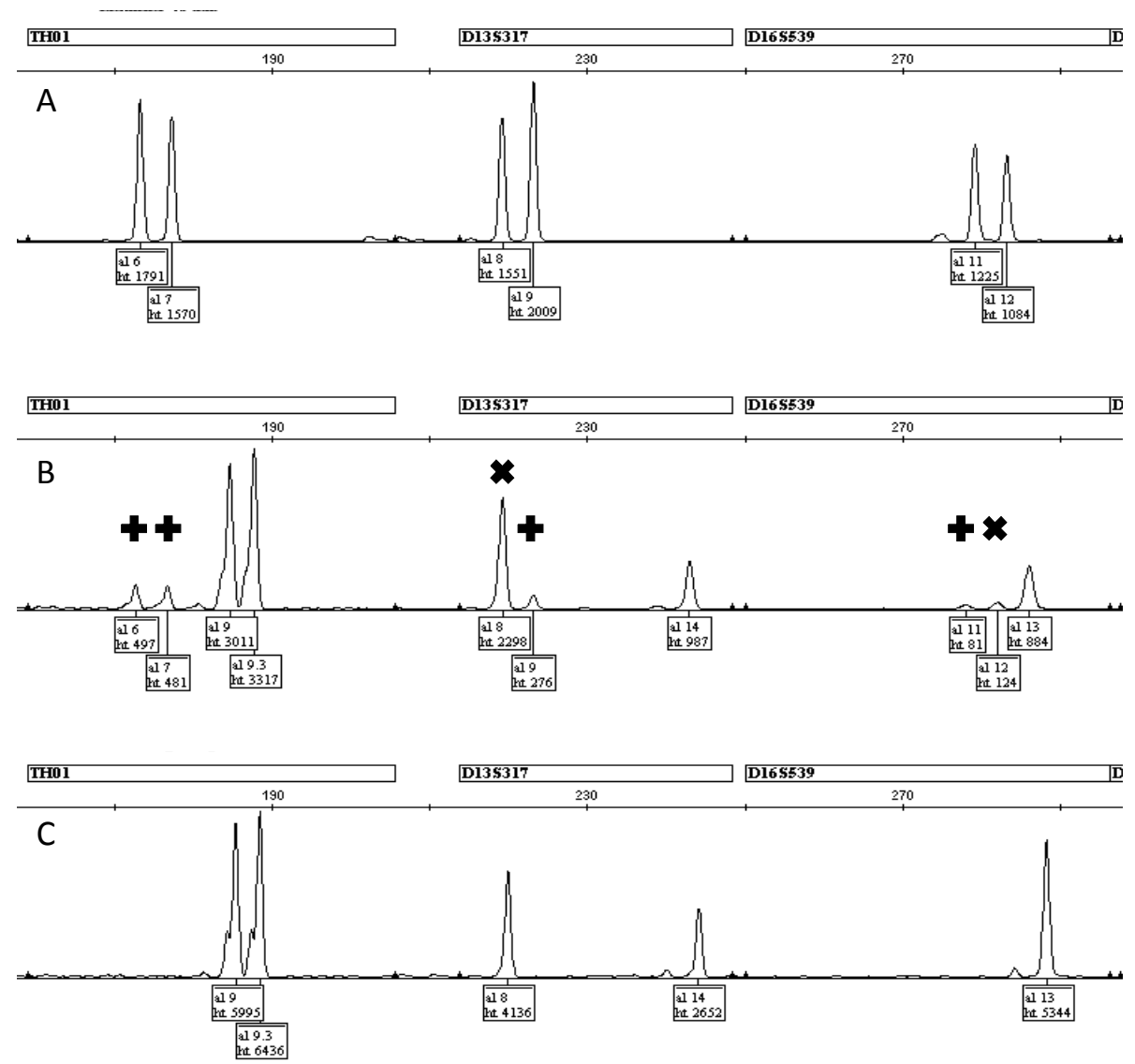

Figure 1: Electropherograms of samples amplification with highlighted alleles which have been used for analysis (represented by + ) and which were excluded from analysis (represented by $\mathbf{x}$ ). Picture represent samples in order - A) male control sample, B) female saliva sample collected in 1 minute after intense kissing, C) female control sample.

male DNA fraction and a-STR profiling with commercially available multiplex PCR a-STR genotyping kit. Moreover, based on current results comparison of performance of different methods (quantitative PCR or multiplex PCR) and different targets (SRY-gene and DYS-gene or Y-STR and a-STR), used in our current and previous study for minor contributor proportion estimation or genotyping in male/female mixed samples, was performed.

\section{Materials and Methods}

\section{Samples}

From all participants informed consent was gained before their samples collection. The a-STR typing was performed on DNA samples originated from the same buccal swab and saliva samples as used in our previous study [10]. Briefly, buccal swab and saliva samples were collected from 12 heterosexual pairs before and after 2 minutes of intense kissing. From men buccal swabs were taken before, and from women whole saliva samples were collected, before and at set time intervals $(1,5,10,30$ and 60 minutes) after intense kissing.

\section{Genotyping}

The AmpFlSTR ${ }^{\circledR}$ Identifiler ${ }^{\circledR}$ Plus PCR Amplification Kit was used for a-STR profiling according to the manufacturer's protocol. Capillary electrophoresis was performed on an ABI-3130xl Genetic Analyzer. To reduce undesired analytical bias introduced by the operator only male alleles different from female ones and outside stutter peak areas of female alleles were investigated and counted if detected (principle of selection of alleles used for analysis is showed in Figure 1). According to this criterion, Counts of Potentially Identifiable Obligatory Alleles (CPIOA, based on genotyping of control male and female samples) and Counts of Identified Obligatory Alleles (CIOA, based on genotyping of mixed salivary samples) were calculated. The success of minor contributor allele identification in our samples was quantified as the CIOA/CPIOA ratio, which was $100 \%$ if all potentially identifiable obligatory alleles were found and $0 \%$ if no potentially identifiable obligatory alleles were found in women's saliva samples. For the estimation of proportion of minor contributor DNA (represented by male DNA), the $\mathrm{Y} / \mathrm{X}$ Amelogenin peak height ratio was used.

\section{Methods and assays comparison}

Additionally, comparison of performance of different methods and assays used in the current and previous study focused on minor contributor male DNA detection in female DNA background was performed [10]. For comparison methods based on quantitative PCR, Y-STR profiling and a-STR profiling were used. Moreover, different assays and ratios represented by assays amplifying SRY and DYS14 gene sequences in quantitative PCR and Y/X and CIOA/CPIOA ratios as parameters in a-STR profiling based calculations were compared. Samples were ranked with respect to their performance in minor contributor male DNA quantitation (quantitative PCR and Y/X ratio) or STR profiling (Y-STR and a-STR genotypes), when the best performing 


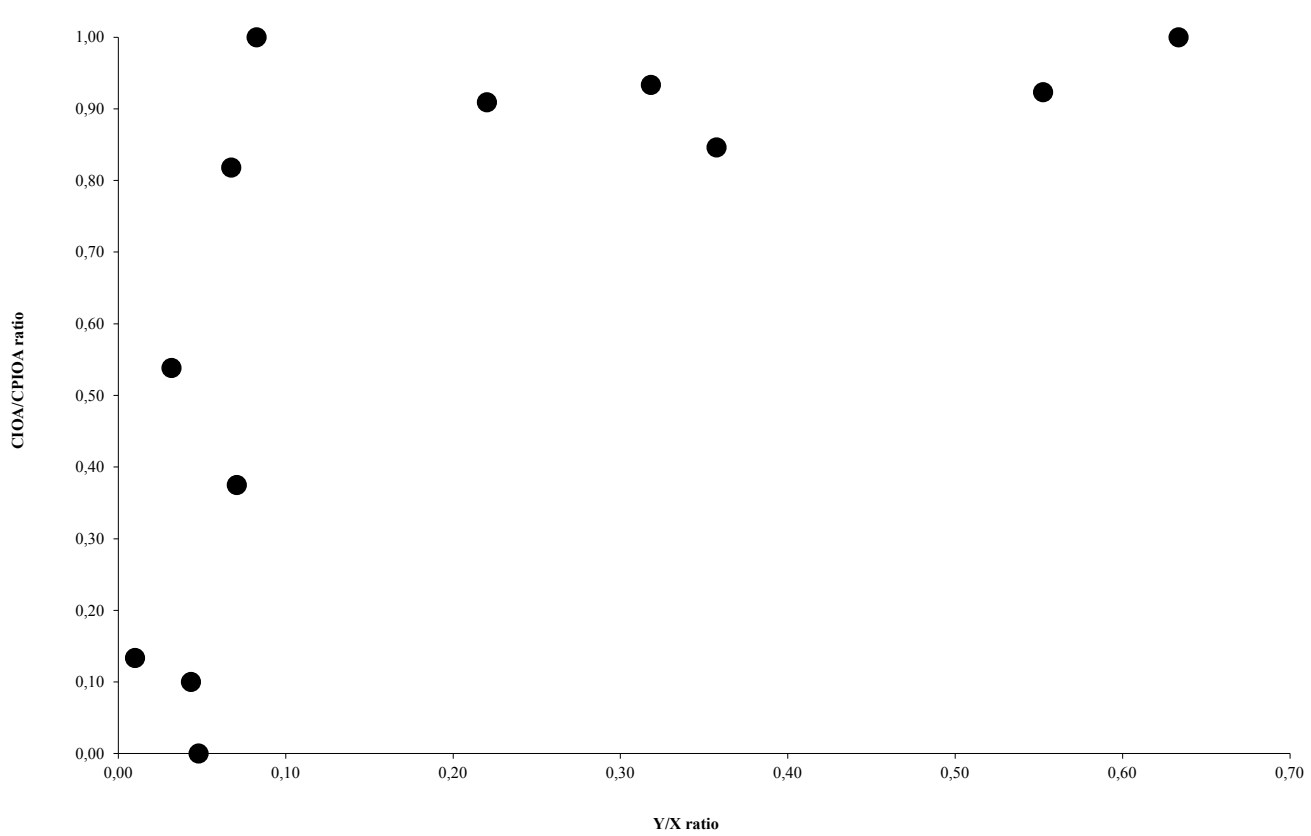

Figure 2: Association between male DNA proportion and successful minor contributor (male) alleles identification presented as relation between $Y / X$ Amelogenin peak height ratio and CIOA/CPIOA ratio.

\begin{tabular}{|c|c|c|c|c|}
\hline Sample & $\mathrm{Y} / \mathrm{X}$ ratio & CIOA* $^{*}$ & $\mathrm{CPIOA}^{\dagger}$ & CIOA/CPIOA ratio \\
\hline A & 0.07 & 9 & 11 & 0.82 \\
\hline B & 0.36 & 12 & 13 & 0.92 \\
\hline C & 0.55 & 13 & 13 & 1.00 \\
\hline D & 0.00 & 2 & 15 & 0.13 \\
\hline$E$ & 0.07 & 3 & 8 & 0.38 \\
\hline $\mathbf{F}$ & 0.63 & 15 & 15 & 1.00 \\
\hline G & 0.22 & 10 & 11 & 0.91 \\
\hline H & 0.32 & 14 & 15 & 0.93 \\
\hline I & 0.04 & 1 & 10 & 0.10 \\
\hline $\mathbf{J}$ & 0.08 & 8 & 8 & 1.00 \\
\hline $\mathbf{K}$ & 0.03 & 7 & 13 & 0.54 \\
\hline $\mathbf{L}$ & 0.05 & 0 & 11 & 0.00 \\
\hline
\end{tabular}

Table 1: Results of genotyping of saliva samples taken 1 minute after intense kissing with AmpFISTR ${ }^{\circledR}$ Identifiler ${ }^{\circledR}$ Plus PCR Amplification Kit. The results are presented as $\mathrm{Y} / \mathrm{X}$ Amelogenin ratio and $\mathrm{CIOA} / \mathrm{CPIOA}$ ratio, which correspond to the male/female ratio and minor contributor allele detection ratio, respectively. ${ }^{*}$ count of identified obligatory alleles; ${ }^{\dagger}$ count of potentially identifiable obligatory alleles.

sample with highest amount of male DNA, highest number of correctly identified alleles or highest ratios was assigned with 1 and the worst performing sample was assigned with 12 .

\section{Results}

\section{STR genotyping of mixed samples}

Even though in control samples all alleles in all 15 tested a-STR markers were genotyped only proportion of them fullfill the criteria used for reduction of the investigator based bias in their correct identification. Therefore in all samples $8-15$ potentially identifiable obligatory alleles (CPIAO) were identified as candidates for use in subsequent CIOA/CPIOA calculations. Samples collected within 1 minute after intense kissing were found to be suitable for a-STR based male fraction calculation and a-STR profiling. In these, the Y/X ratio varied between $0 \%$ and $63 \%$ and at least some correct male alleles (CIOA) were identified in 11 of 12 samples. The CIOA/CPIOA ratio varied from $0 \%$ to $100 \%$ and it was found to be associated with male
DNA proportion estimated by the $\mathrm{Y} / \mathrm{X}$ ratio, where in samples with a Y/X ratio higher than $7 \%$, more than $80 \%$ of potentially identifiable alleles were found (Figure 2). Detailed information about male DNA relative quantification, represented by $\mathrm{Y} / \mathrm{X}$ ratio calculations, and minor contributor a-STR profiling success, represented by CIOA/CPIOA ratio, in all samples is summarised in Table 1. From all samples collected 5 minutes after kissing, only in one mixed profile with obligatory male alleles was identified (sample $\mathrm{C}$ ). The $\mathrm{Y} / \mathrm{X}$ ratio of this sample reached $15 \%$ and CIOA/CPIOA ratio was $77 \%$. In samples collected 10,30 and 60 minutes after kissing no Y-chromosomal signal and none of the potentially identifiable obligatory alleles were identified.

\section{Methods and assays comparison}

After the comparison of results between our previously published [10] and currently presented study, we have recorded consistent performance of different methods for the analysis of male/female mixtures. Summarized data from the comparison are presented in Table 2. 
Page 4 of 5

\begin{tabular}{|c|c|c|c|c|c|}
\hline Sample & SRY* & DYS* & Y-STR $^{\dagger}$ & $\mathrm{Y} / \mathrm{X}$ ratio $\neq$ & CIOA/CPIOA ratio $\ddagger$ \\
\hline A & 6 & 8 & 9 & 8 & 7 \\
\hline B & 5 & 5 & 5 & 3 & 5 \\
\hline C & 2 & 4 & 2 & 2 & 2 \\
\hline D & 7 & 7 & 3 & 12 & 10 \\
\hline$E$ & 4 & 2 & 6 & 7 & 9 \\
\hline $\mathbf{F}$ & 1 & 1 & 1 & 1 & 1 \\
\hline G & 8 & 6 & 8 & 5 & 6 \\
\hline $\mathbf{H}$ & 3 & 3 & 4 & 4 & 4 \\
\hline I & 12 & 11 & 11 & 10 & 11 \\
\hline $\mathbf{J}$ & 10 & 9 & 10 & 6 & 3 \\
\hline $\mathbf{K}$ & 11 & 12 & 12 & 11 & 8 \\
\hline $\mathbf{L}$ & 9 & 10 & 7 & 9 & 12 \\
\hline
\end{tabular}

Table 2: Summary of performance of samples with all tested methods and targets from our previously published and current study. Results from individual assays were converted to rank where the best performing sample was assigned with 1 and the worst performing with $12 .{ }^{*}$ qPCR-based assays, ${ }^{\dagger}$ AmpFISTR ${ }^{\circledR}$ Yfiler ${ }^{\circledR}$ PCR Amplification Kit-based genotyping, ${ }^{\ddagger}$ AmpFlSTR ${ }^{\circledR}$ Identifiler $^{\circledR}$ Plus PCR Amplification Kit-based genotyping.

\section{Discussion}

Genetic characterisation of unbalanced mixed stains remains an important topic in forensic science where improvement is needed. Mixed sample resolution based on Y-STR analysis in male/female mixed samples is relatively straightforward but its applicability in direct personal identification is limited [7]. In our previous study based on Y-chromosome sequence identification and Y-STR profiling we showed that salivary samples gained from females could be a useful source of forensic evidence in specific cases of sexual assaults in which the victim (female) was constrained to kissing by the perpetrator (male) [10]. In the current study, we tested if it is possible to utilize a-STR genotyping analysis on the same sample set. It is well known that resolution of mixed samples after a-STR analysis is one of the main challenges which complicate routine and automated analysis of biological traces in forensics [11]. The main problems associated with mixtures are: the low amount of isolated DNA from forensic traces; inappropriate quality of isolated DNA; unknown number of contributors in the sample; contributor(s)' genotype composition; availability of reference samples; and proportion of minor contributor DNA [12-16]. In our study, fresh salivary or buccal swab samples of known contributors were analysed, thus some of the issues mentioned above were not applicable.

To avoid subjective bias and to minimise the influence of contributor genotype composition, the success of analysis was evaluated with use of the CIOA/CPIOA ratio. This method for minor contributor DNA analysis was chosen because our study was focused on technical aspects of utilisation of a-STR profiling for mixed samples genotyping. Therefore standard calculations of random match probability of identified genotype were not performed. Moreover, these calculations will be strongly dependent on DNA profiles of both contributors in DNA samples when same criteria as in our study will be used in real samples analysis, therefore such calculations based on our sample group will not be universally applicable. For relative estimation of proportion of minor contributor DNA (in male/female mixtures), the Y/X ratio was used. This parameter is accepted as a standard marker for the presence of male DNA in mixed samples as well as a parameter representing the level of male DNA in male/female mixtures [17]. In our samples, the $\mathrm{Y} / \mathrm{X}$ ratio varied between $0 \%$ and $63 \%$, which is in concordance with results of estimation of this ratio in pure male DNA samples, where it was found to vary between $80 \%$ and $136 \%$ [18]. After analysing the possible association of success of a-STR analysis (represented by CIOA/ CPIOA ratio) and the level of male DNA identified in salivary samples (represented by Y/X ratio), we were able to identify the association of the Y/X ratio and the outcome of a-STR profiling. It could be seen that samples with $\mathrm{Y} / \mathrm{X}$ ratio higher than $7 \%$ resulted in relevant a-STR profiling for personal identification in forensics; hence, more than $80 \%$ of potentially identifiable obligatory alleles could be identified (Table 1 and Figure 2). However, to confirm this statement more rigorous analysis of artificially prepared samples with different a-STR profiling kits should be performed in future. Nevertheless, our experimental findings are consistent with the results of artificially prepared mixture samples with specific PowerPlex ${ }^{\star}$ kit-based analysis, where artificially prepared mixtures with $5 \%$ of minor contributor DNA enabled the identification of 52-95\% of unique minor contributor alleles [8].

When comparing our data with the data of Banaschak et al. [9], we were able to confirm previous findings that a-STR profiling assays are sensitive enough to identify minor contributor alleles in samples collected immediately after the kissing. Moreover, according to our results in small proportion of cases the minor contributor male DNA can be suitable for a-STR profiling also 5 minutes after kissing. As could be expected no minor contributor a-STR alleles were detected in samples collected 10, 30 and 60 minutes after intense kissing. The continuing salivation of the women diluted the male specimen below the detection limit of a-STR profiling method based on multiplex PCR and capillary electrophoresis. Therefore, saliva or mouthwash samples could be used for standard PCR based a-STR profiling, especially in cases when women did not survive the sexual attack or if they survived and spat saliva immediately after constrained kissing onto material which can conserve DNA for a much longer time. In murder cases when (constrained) kissing could be considered and the perpetrator manipulated the corpse of victim after the act, saliva or mouthwash samples could be used. This is also true if the perpetrator cleaned the victim's body (e.g. area of lips) or crime scene or removed the clothing of the victim where the perpetrator specimen can usually be found.

Finally, we performed a comparison of different methods and targets used for detection of the presence of male DNA and the individual profiling used in our previous and current study. This comparison showed that the same samples could be analysed using different methods obtaining similar results, but with different levels of sensitivity, although few exceptions were recorded (Table 2, samples $\mathrm{D}$ and $\mathrm{E}$ ). Using $\mathrm{qPCR}$, it was possible to determine the presence of male DNA in the best performing salivary samples up to 10 or 60 minutes after kissing, depending on the assay (target) used. Using Y-STR profiling, the full profile could be identified up to 30 minutes after kissing and with a-STR profiling, the sample can be successfully genotyped if collected up to 5 minutes after kissing. 
Citation: Repiská G, Durdiaková J, Kamodyová N, Minárik G (2015) The Applicability of Autosomal Short Tandem Repeats Genotyping for Minor Contributor DNA Profiling from Mixed Saliva Samples. J Forensic Res 6: 259. doi:10.4172/2157-7145.1000259

Page 5 of 5

In conclusion, we showed that salivary samples collected after constrained kissing of a living victim have limited applicability for perpetrator a-STR profiling and confirmed that our previously and currently presented results are in agreement. Routine methods which are currently used for a-STR profiling based on standard PCR and capillary electrophoresis analysis have reached their sensitivity levels, while low levels mixtures remain problematic for comprehensive forensic analysis. But new methods of forensic analysis of mixed samples based on next generation sequencing, which are currently in phase of testing and implementation, will significantly improve sensitivity without loss of specificity of analysis $[19,20]$. This could lead to redefining of many analytical criteria for the successful identification of the perpetrator's DNA profile, not strictly based only on STR profiling as SNP or DIP analysis could be also applied.

\section{Acknowledgement}

This work was supported by the Slovak Research and Development Agency through contracts VMSP-II-0030-09 and APVV-0720-10.

\section{Declaration of Authorship}

GR performed aSTR profiling using capillary electrophoresis and analysed the results. Also she participated in writing of the manuscript. JD and NK collected the samples and isolated DNA. GM designed the study. He participated in analysing the results and writing of the manuscript.

\section{References}

1. Torres Y, Flores I, Prieto V, López-Soto M, Farfán MJ, et al. (2003) DNA mixtures in forensic casework: a 4-year retrospective study. Forensic Sci Int 134: 180-186.

2. Gill P, Brenner CH, Buckleton JS, Carracedo A, Krawczak M, et al. (2006) DNA commission of the International Society of Forensic Genetics: Recommendations on the interpretation of mixtures. Forensic Sci Int 160: 90101.

3. Vuichard S, Borer U, Bottinelli M, Cossu C, Malik N, et al. (2011) Differential DNA extraction of challenging simulated sexual-assault samples: a Swiss collaborative study. Investig Genet 2: 11

4. Cerri N, Ricci U, Sani I, Verzeletti A, De Ferrari F (2003) Mixed stains from sexual assault cases: autosomal or Y-chromosome short tandem repeats? Croat Med J 44: 289-292.

5. Roewer L (2009) Y chromosome STR typing in crime casework. Forensic Sci Med Pathol 5: 77-84.
6. de Knijff P (2003) Son, give up your gun: presenting Y-STR results in court Profiles in DNA 6: 3-5.

7. Hanson EK, Ballantyne $\mathrm{J}$ (2007) An ultra-high discrimination $Y$ chromosome short tandem repeat multiplex DNA typing system. PLoS One 2: e688.

8. Tucker VC, Hopwood AJ, Sprecher CJ, McLaren RS, Rabbach DR, et al. (2012) Developmental validation of the PowerPlex(R) ESX 16 and PowerPlex(R) ESX 17 Systems. Forensic Sci Int Genet 6: 124-131.

9. Banaschak S, Möller K, Pfeiffer H (1998) Potential DNA mixtures introduced through kissing. Int J Legal Med 111: 284-285.

10. KamodyovÃ N, DurdiakovÃ J, Celec P, SedlÃ Ä kovÃ T, Repiskã G, et al. (2013) Prevalence and persistence of male DNA identified in mixed saliva samples after intense kissing. Forensic Sci Int Genet 7: 124-128.

11. Budowle B, Onorato AJ, Callaghan TF, Della Manna A, Gross AM, et al. (2009) Mixture interpretation: defining the relevant features for guidelines for the assessment of mixed DNA profiles in forensic casework. J Forensic Sci 54 810-821.

12. Van Oorschot Ra, Ballantyne Kn, Mitchell Rj (2010) Forensic Trace Dna: A Review. Investig Genet 1: 14

13. Ludes B, Keyser C (2011) Avoiding Errors and Pitfalls in Evidence Sampling for Forensic Genetics, InTech, Rijeka.

14. Perlin MW, Legler MM, Spencer CE, Smith JL, Allan WP, et al. (2011) Validating TrueAllele® DNA mixture interpretation. J Forensic Sci 56: 1430-1447.

15. Pfeifer CM, Klein-Unseld R, Klintschar M, Wiegand P (2012) Comparison of different interpretation strategies for low template DNA mixtures. Forensic Sci Int Genet 6: 716-722

16. Haned H, Pène L, Lobry JR, Dufour AB, Pontier D (2011) Estimating the Number of Contributors to Forensic DNA Mixtures: Does Maximum Likelihood Perform Better Than Maximum Allele Count? J Forensic Sci 56: 23-28.

17. Butler JM (2012) Y-Chromosome DNA testing., Academic Press, San Diego.

18. Gilder JR, Inman K, Shields W, Krane DE (2011) Magnitude-dependent variation in peak height balance at heterozygous STR loci. Int J Legal Med 125: 87-94.

19. Holland MM, McQuillan MR, O'Hanlon KA (2011) Second generation sequencing allows for mtDNA mixture deconvolution and high resolution detection of heteroplasmy. Croat Med J 52: 299-313.

20. Debeljak M, Freed DN, Welch JA, Haley L, Beierl K, et al. (2014) Haplotype counting by next-generation sequencing for ultrasensitive human DNA detection. J Mol Diagn 16: 495-503. 